\title{
Deber rationale Verbindungen der elliptischen Transcendenten.
}

Jede rationale Funktion von

$$
\sin \varphi, \quad \cos \varphi, \quad \Delta \varphi=\sqrt{1-k^{2} \sin ^{2} \varphi}
$$

wird sich aus einer ganzen Funktion dieser Grössen und ans einer Summe von Gliedern zusammensetzen lassen, deren jedes die Form:

$$
\frac{A+B \cos \varphi+C \Delta \varphi+D \cos \varphi \Delta \varphi}{(a+b \sin \varphi)^{ \pm n}}
$$

hat, worin $n$ eine positive ganze Zahl ist und die Grössen $A, B, C, D, a, b$ von der Variabeln $\varphi$ unabhängig sind.

Der genannte Ausdruck ist daher die allgemeine Form jeder gebrochenen Funktion der angegehenen Grössen. Ein besonderer Fall, der in der Bestimmung von $a$ und $b$ liegt, ist die Form:

$$
\sin \varphi^{ \pm p} \cdot \cos \varphi^{ \pm r} \cdot \Delta \varphi^{ \pm s}
$$

worin $p, r, s$ positive ganze Zahlen bedeuten.

Die elliptischen Funktionen $\sin \varphi, \cos \varphi, \Delta \varphi$ hängen bekanntlich von den Variabeln $q$ und $x$ ab, so dass $\varphi=a m \frac{2 K x}{T}=a m u$ und Jacobis Fundamente enthalten Reihen, die sowohl diese Funktionen als deren ganze Potenzen und Produkte auf die verschiedenste Weise nach $q$ und $x$ darstellen. Wird das Produkt

$$
\sin \varphi^{ \pm p} \cdot \cos \varphi^{ \pm r} \cdot \Delta \varphi^{ \pm s}
$$

mit $Z(x)$ multiplizirt, worin nach Fnnd. $\$ .47$ ist:

$$
Z(x)=\left(\frac{2 K}{\pi}-\frac{2 E}{\pi}\right) x-\frac{2 K}{\pi} \int_{0}^{x} k^{2} \sin ^{2} a m \frac{2 K x}{\pi} d x,
$$


so habe ich in 37. Bande von Crelles Journal für Mathematik die Entwickelung dieses Ausdrucks nach den Sinus und Cosinus der Vielfachen von $x$ gegeben. Es hängt dieselbe von der Differentiation und Integration bekannter Reihen für $\sin x, \cos x, \Delta \varphi, Z(x)$ nach $x$ und von der einmaligen Differentiation derselben Reihen nach $q$ ab, welche Operationen sich ohne Schwierigkeit ausführen lassen, sobald die Form der Reihen gewählt wird, in der die Sinus und Cosinus der Vielfachen von $x$ vorkommen.

Im Folgenden soll sowohl

$$
\frac{A+B \cos \varphi+C \Delta \varphi+D \cos \varphi \Delta \varphi}{(a+b \sin \varphi)^{n}}
$$

als auch das Produkt dieses Ausdrucks mit $Z(x)$ anf dieselbe Weise entwickelt werden; wohei sich ein dem Vorigen ähnliches Resultat herausstellen wird. In dem ersten Abschnitt werden die Grundformen aufgesucht werden, auf welche die übrigen sich zurückführen lassen. Der zweite Abschnitt enthält die Reihenentwickelungen dieser Grundformen.

\section{Erster Abschnitt.}

In der allgemeinen Form:

$$
\frac{A+B \cos \varphi+C \Delta \varphi+D \cos \varphi \Delta \varphi}{(a+b \sin \varphi)^{n}}
$$

kann, insofern der früher besprochene Fall *) nicht eintritt, der Koefficient $a$ anf 1 gebracht werden, so dass ich sogleich zu dem Ausdrucke

$$
\frac{A+B \cos \varphi+C \Delta \varphi+D \cos \varphi \Delta \varphi}{(1+b \sin \varphi)^{n}}
$$

übergehe, worin $A, B, C, D, b$ Funktionen von $g$ und $\varphi=a m(u)=a m \frac{2 K x}{\pi}$ als Funktion von $x$ und $q$ zu betrachten sind; $n$ dagegen eine ganze positive Zahl ist. Bezeichnet man einen solchen Ausdruck ganz abgesehn von der Grösse der Koefficienten $A, B, C, D$, $b$, nur in Rücksicht darauf, dass er den Exponenten $n$ enthält, mit $U_{n}$, und also mit $U_{n-1}, U_{n-2}$, $\ldots U_{1}$, sobald der Index die Werthe $n-1, n-2, \ldots 1$ annimmt, so lässt sich der Exponent $n$ durch Differentiation nach $u$ stets auf die Einheit herabbringen. Da nämlich

$$
d \sin \varphi=\cos \varphi \Delta \varphi d u, d \cos \varphi=-\sin \varphi \Delta \varphi d u, d \Delta \varphi=-k^{2} \sin \varphi \cos \varphi d u
$$

ist, so kann der Differentialquotient von $U_{n-1}$ nach $u$ nur Glieder enthalten, die die Form $U_{n}$, oder $U_{p}$ baben, worin $p<n$ ist; folglich lässt sich $U_{n}$ durch $U_{n-1} U_{n-2} \ldots$ und $d \dot{U}_{n-1}: d u$ ausdrücken. Durch Vertauschung der Indices führt $U_{n-1}$ auf $U_{n-2}, U_{n-3} \ldots$ $d U_{n-2}: d u$, so dass schliesslich der Ausdruck $U_{n}$ nur $U_{1}$, dessen mehrmalige Differential-

*) $\sin \varphi \pm p \cdot \cos \varphi \pm r \cdot \Delta \varphi \pm s$ 
quotienten nach $u$, und endlich ganze Funktionen von $\sin \varphi, \cos \varphi, \Delta \varphi$ enthält. Es bleibt demnach nur die Funktion $U_{1}$

$$
\frac{A+B \cos \varphi+C \Delta \varphi+D \cos \varphi \Delta \varphi}{1+b \sin \varphi}
$$

die verschiedenen Differentialquotienten von $U_{1}$ nach $u$ und das Produkt derselben mit $Z(x)$ zur nähern Betrachtung übrig.

Wird die Entwickelung von $V_{1}$ als bekannt vorausgesetzt, fortgehend nach den Sinus und Cosinus der Vielfachen von $x=\frac{u \pi}{2 K}$, so lassen sich die Differentialquotienten von $U_{1}$ nach $u$ leicht ableiten. Ebenso aber lässt sich übersehn, dass das Produkt eines jeden derselben mit $Z(x)$ oder $\frac{d^{p} U_{1}}{d u^{p}} Z(x)$ durch Differentiation von $U_{1}$ und $U_{1} \cdot Z(x)$ abgeleitet werden kann. Es ist nämlich:

Da aher ist:

$$
\frac{d \frac{d^{p-1} U_{1}}{d u^{p-1}} Z(x)}{d u}=\frac{d U_{1}^{p}}{d u^{p}} Z(x)+\frac{d^{p-1} U_{1}}{d u^{p-1}} \cdot \frac{d Z(x)}{d u}
$$

$$
\frac{d Z(x)}{d u}=1-\frac{E^{\prime}}{K}-k^{2} \sin ^{2} \varphi
$$

so folgt:

$$
Z(x) \frac{d^{p} U_{1}}{d u^{p}}=\frac{d \frac{d^{p-1} U_{1}}{d u^{p-1}} Z(x)}{d u}-\frac{d^{p-1} U_{1}}{d u^{p-1}}\left\{1-\frac{E^{\prime}}{K}-k^{2} \sin ^{2} \varphi_{\}}\right.
$$

Das zweite Glied der rechten Seite ist eine rationale Funktion von $\sin \varphi, \cos \varphi, \Delta \varphi$, während das erste Glied derselben Seite nur den $(p-1)$ ten Differentialquotienten von $U_{1}$ enthält. Wiederholt man dieses Verfahren, so erlangt man für $\frac{d^{p} U_{1}}{d u^{p}} Z(x)$ endlich einen Ausdruck, der aus zwei Theilen besteht; der eine ist $\frac{d^{p}\left(U_{1} Z(x)\right)}{d u^{p}}$, und der zweite ist eine rationale Funktion von $\sin \varphi, \cos \varphi, \Delta \varphi$, die auf die Form $U_{1}$ gebracht werden kann.

Es bleibt noch $U_{1}$ und $Z(x) U_{1}$, woranf alle Funktionen, die hier in Betrachtung gezogen worden, sich zurückführen lassen, zu entwickeln übrig. In dem Ausdrucke $U_{1}$ ist $b$ im Allgemeinen eine Funktion von $q$. Man bringe $b$ unter die Form $k \sin a m(a)$ und setze am $(a)=\alpha$, so dass $b=k \sin \alpha$ und sowohl $\varphi$ als $\alpha$ von demselben Modul $k$ abhängen; dann wird:

dessen 4 Glieder:

$$
U_{1}=\frac{A+B \cos \varphi+C \Delta \varphi+D \cos \varphi \Delta \varphi}{1+k \sin \alpha \sin \varphi}
$$

$$
\frac{1}{1+k \sin \alpha \sin \varphi}, \frac{\cos \varphi}{1+k \sin \alpha \sin \varphi}, \frac{\Delta \varphi}{1+k \sin \alpha \sin \varphi}, \frac{\Delta \varphi \cos \varphi}{1+k \sin \alpha \sin \varphi}
$$

nun getrennt untersucht werden sollen, abgesehn von ihren Koefficienten. 
Der letztere der 4 Ausdrücke führt auf den ersten. Denn bildet man den Differentialquotienten des vierten nach $u$, so wird:

$$
\begin{gathered}
\frac{d \frac{\Delta \varphi \cos \varphi}{1+k \sin \alpha \sin \varphi}}{d u}=A_{1}(1+k \sin \alpha \sin \varphi)^{2}+A_{2}(1+k \sin \alpha \sin \varphi)+A_{3} \\
\frac{A_{4}}{1+k \sin \alpha \sin \varphi}+\frac{A_{3}}{(1+k \sin \alpha \sin \varphi)_{2}}
\end{gathered}
$$

worin $A_{1}, A_{2}, A_{3}, A_{4}, A_{5}$ Funktionen von $k$ und $\sin \alpha$ sind. Ferner sind

$$
\frac{d \frac{1}{1+k \sin \alpha \sin \varphi}}{d a}=\frac{\cos \alpha \Delta \alpha}{\sin \alpha} \cdot \frac{1}{(1+k \sin \alpha \sin \varphi)^{2}}-\frac{\cos \alpha \Delta \alpha}{\sin \alpha} \cdot \frac{1}{1+k \sin \alpha \sin \varphi}
$$

oder

$$
\frac{1}{(1+k \sin \alpha \sin \varphi)^{2}}=\frac{1}{1+k \sin \alpha \sin \varphi}+\frac{\sin \alpha}{\cos \alpha \cdot \Delta \alpha} \cdot \frac{d+k \sin \alpha \sin \varphi}{d \alpha}
$$

wodurch entsteht

$$
\begin{aligned}
\frac{d \frac{\Delta \varphi \cos \varphi}{1+k \sin \alpha \sin \varphi}}{d u}=A_{1}(1+k \sin \alpha \sin \varphi)^{2}+A_{2}(1+k \sin \alpha \sin \varphi)+A_{3} \\
+\frac{A_{4}+A_{5}}{1+k \sin \alpha \sin \varphi}+A_{3} \frac{d \frac{1}{1+k \sin \alpha \sin \varphi}}{d a} \cdot \frac{\sin \alpha}{\cos \alpha \Delta \alpha}
\end{aligned}
$$

Es führt also der Differentialquotient nach $u$ von:

$$
\frac{\Delta \varphi \cos \varphi}{1+k \sin \alpha \sin \varphi}
$$

auf ganze Funktionen von $\varphi$, ausserdem noch auf $1:(1+k \sin \alpha \sin \varphi)$ und dessen Differentialquotient nach $a$, wofür auch der nach $u$ gesetzt werden kann. nach $u$ gelangt man zu $\Delta \varphi \cos \varphi:(1+k \sin \alpha \sin \varphi)$. Endlich ist noch

zu behandeln, wofür man auch

$$
\frac{\Delta \varphi \cdot \cos \varphi Z(x)}{1+k \sin \alpha \sin \varphi}
$$

$$
\frac{\Delta \varphi \cdot \cos \varphi}{1+k \sin \alpha \sin \varphi} \int_{0}^{u} \sin ^{2} \varphi d u=P
$$

wählen kann. Es ist hievan der Differentialquotient nach $u$,

$$
\begin{gathered}
\frac{d P}{d u}= \\
\frac{d \frac{\Delta \varphi \cos \varphi}{1+k \sin \alpha \sin \varphi}}{d u} \int_{0}^{u} \sin ^{2} \varphi d u+\frac{\Delta \varphi \cos \varphi \sin ^{2} \varphi}{1+k \sin \alpha \sin \varphi}
\end{gathered}
$$


Setzt man für $\frac{d \frac{\Delta \varphi \cos \varphi}{1+k \sin \alpha \sin \varphi}}{d u}$ den vorhin gefundenen Werth ein, so blieben ausser den behandelten Formen noch übrig

$$
\frac{1}{1+k \sin \alpha \sin \varphi} \int \sin ^{2} \varphi d u \text { und } \frac{d \frac{1}{1+k \sin \alpha \sin \varphi}}{d u} \int \sin ^{2} \varphi d u
$$

wovon die zweite durch Differentiation der erstern abgeleitet werden kann. Demnach bleibt für den zweiten Abschnitt nur übrig die Reihenentwickelung für

$$
\begin{array}{ccc}
\frac{1}{1+k \sin \alpha \sin \varphi}, & \frac{\Delta \varphi}{1+k \sin \alpha \sin \varphi}, & 1+k \cos \varphi \\
\frac{Z(x)}{1+k \sin \alpha \sin \varphi}, & \frac{Z(x) \Delta \varphi}{1+k \sin \alpha \sin \varphi}, & \frac{Z(x) \cdot \cos \varphi}{1+k \sin \alpha \sin \varphi} .
\end{array}
$$

\section{Zweiter Abschnitt.}

Die bekannten Formeln für die Addition und Subtraktion der elliptischen Funktionen sind:

$$
\begin{aligned}
\sin \sigma & =\frac{\sin \varphi \cos \alpha \Delta \alpha+\sin \alpha \cos \varphi \Delta \varphi}{1-k^{2} \sin ^{2} \alpha \sin ^{2} \varphi} \\
\cos \sigma & =\frac{\cos \varphi \cdot \cos \alpha-\sin \varphi \sin \alpha \Delta \varphi \Delta \alpha}{1-k^{2} \sin ^{2} \alpha \sin ^{2} \varphi} \\
\Delta \sigma & =\frac{\Delta \varphi \cdot \Delta \alpha-k^{2} \sin \varphi \sin \alpha \cos \varphi \cdot \cos \alpha}{1-k^{2} \sin ^{2} \alpha \sin ^{2} \varphi} \\
\sin \delta & =\frac{\sin \varphi \cos \alpha \Delta \alpha-\sin \alpha \cos \varphi \Delta \varphi}{1-k^{2} \sin ^{2} \alpha \sin ^{2} \varphi} \\
\cos \delta & =\frac{\cos \varphi \cdot \cos \alpha+\sin ^{2} \varphi \sin ^{2} \Delta \varphi \Delta \alpha}{1-k^{2} \sin ^{2} \alpha \sin { }^{2} \varphi} \\
\Delta \delta & =\frac{\Delta \varphi \cdot \Delta \alpha+k^{2} \sin ^{2} \sin \alpha \cos \varphi \cdot \cos \alpha}{1-k^{2} \sin ^{2} \alpha \sin { }^{2} \varphi}
\end{aligned}
$$

worin $\varphi=a m(u), \alpha=a m(a), \sigma=a m(u+a), d=a m(u-a)$ gesetzt ist. Aus diesen Formeln folgt:

$$
\begin{aligned}
& k \cos \sigma+k \cos \delta+\Delta \sigma-\Delta \delta=\frac{2 k \cos \alpha \cos \varphi}{1+k \sin \alpha \sin \varphi} \\
& \Delta \sigma+\Delta \delta+k \cos \sigma-k \cos \delta=\frac{2 \Delta \alpha \cdot \Delta \varphi}{1+k \sin \alpha \sin \varphi}
\end{aligned}
$$


und hieraus:

$$
\begin{aligned}
\frac{\cos \varphi}{1+k \sin \alpha \sin \varphi} & =\frac{k \cos \sigma+k \cos \delta+\Delta \sigma-\Delta^{\prime} \delta}{2 k \cos \alpha} \\
\frac{\Delta \varphi}{1+k \sin \alpha \sin \varphi} & =\frac{\Delta \sigma+\Delta \delta+k \cos \sigma-k \cos \delta}{2 \Delta \alpha}
\end{aligned}
$$

Nimmt man noch zu Hilfe aus dem 53. Kapitel der Fundamente die Formel

so folgt:

$$
2 Z(a)+Z(u-a)-Z(u+a)=\frac{2 k^{2} \sin \alpha \cos \alpha \Delta \alpha \sin ^{2} \varphi}{1-k^{2} \sin ^{2} \alpha \sin ^{2} \varphi}
$$

$$
k \sin \sigma+k \sin \delta-2 Z(a)-Z(u-a)+Z(u+a)=\frac{2 k \cos \alpha \Delta \alpha \sin \varphi}{1+k \sin \alpha \sin \varphi}
$$

oder

$$
\frac{k \sin \alpha \sin \varphi}{1+k \sin \alpha \sin \varphi}=\frac{\sin \alpha}{2 \cdot \cos \alpha \Delta \alpha}\{k \sin \sigma+k \sin \delta-2 Z(a)-Z(u-a)+Z(u+a)\}
$$
und da

ist, so ergiebt sich

$$
\frac{1}{1+k \sin \alpha \sin \varphi}=1-\frac{k \sin \alpha \cdot \sin \varphi}{1+k \sin \alpha \sin \varphi}
$$

$$
\frac{1}{1+k \sin \alpha \sin \varphi}=1-\frac{2 \cos \alpha \Delta \alpha}{\sin \alpha}\{k \sin \sigma+k \sin \delta-2 Z(a)-Z(u-a)+Z(u+a)\}
$$

Führt man statt $k \sin \alpha$ wiederum $b$ ein, so wird

$$
\begin{aligned}
& \text { 1... } \frac{\cos \varphi}{1+b \sin \varphi}=\frac{1}{2 \sqrt{k^{2}-b^{2}}}\{k \cos \sigma+k \cos \delta+\Delta \sigma-\Delta \delta\} \\
& 2 \ldots \frac{\Delta \varphi}{1+b \sin \varphi}=\frac{1}{2 \sqrt{1-b^{2}}}\{\Delta \sigma+\Delta \delta+k \cos \sigma-k \cos \delta\} \\
& 3 \ldots \frac{\sin \varphi}{1+b \sin \varphi}=\quad \frac{1}{2 \sqrt{1-b^{2}} \sqrt{k^{2}-b^{2}}}\{k \sin \sigma+k \sin \delta-2 Z(a)-Z(u-a)+Z(u+a)\} \\
& \text { 4... } \frac{1}{1+b \sin \varphi}=1-\frac{b}{2 \sqrt{1-b^{2}} \sqrt{k^{2}-b^{2}}}\{k \sin \sigma+k \sin \delta-2 Z(a)-Z(u-a)+Z(u+a)\}
\end{aligned}
$$

Es bleiben noch die Funktionen:

zu entwickeln übrig.

$$
\frac{\cos \varphi Z(u)}{1+b \sin \varphi}, \quad \frac{\Delta \varphi Z(u)}{1+b \sin \varphi}, \frac{\sin \varphi Z(u)}{1+b \sin \varphi}
$$


Nach der üblichen Bezeichnung ist:

$$
\begin{aligned}
u & =\frac{2 K x}{\pi}, \cos \varphi=\sqrt{\frac{\bar{k}}{k}} \cdot \frac{H\left(k+\frac{1}{2} \pi\right)}{\Theta(x)}, \sin \varphi=\frac{1}{\sqrt{k}} \cdot \frac{H(x)}{\Theta(x)} \\
\Delta \varphi & =\sqrt{\overline{k^{\prime}}} \cdot \frac{\Theta\left(x+\frac{1}{2} \pi\right)}{\Theta(x)}, \sqrt{k}=\frac{H\left(\frac{1}{2} \pi\right)}{\Theta\left(\frac{1}{2} \pi\right)}, \sqrt{\left.k^{\prime}\right)}=\frac{\Theta\left(\frac{1}{2} \pi\right)}{\Theta(o)}, \sqrt{\frac{2 K}{\pi}}=\Theta\left(\frac{1}{2} \pi\right)
\end{aligned}
$$

Ferner gleichbedeutend $Z(u)$ mit $Z(x)$; so dass:

$$
\begin{gathered}
\frac{2 K}{\pi} Z(x)=\frac{2 K}{\pi} Z(u)=\frac{\Theta^{\prime}(x)}{\Theta(x)}=\int_{0}^{x}\left(\frac{2 K}{\pi}\right)^{2} \Delta^{2} \varphi d x-\frac{2 K}{\pi} \cdot \frac{2 E^{\prime}}{\pi} \alpha \\
\frac{d \frac{2 K}{\pi} Z}{d x}=\left(\frac{2 K}{\pi}\right)^{2} \Delta{ }^{2} \varphi-\frac{2 K}{\pi} \cdot \frac{2 E^{\prime}}{\pi} .
\end{gathered}
$$

Ich werde die Formeln zusammenstellen, welche für das Folgende gebraucht werden und die theils die Differentialquotienten einzelner Grössen nach $x$ und $q$ sind, theils Relationen zwischen den Konstanten (Funktionen von $q$ ) angeben; deren Richtigkeit leicht erwiesen werden kaun. Ist die Differentiation durch Indices angegeben, so bezieht sie sich auf $x$, so dass z. B. $H^{\prime \prime}\left(\frac{1}{2} \pi\right)$ bedeutet, dass in $\frac{d^{2} H\left(x+\frac{1}{2} \pi\right)}{d x^{2}}$ nach der Differentiation nach $x$, für $x$ Null gesefzt werden soll.

$$
\begin{aligned}
& \frac{2 K}{\pi} \cdot \frac{2 E^{\prime}}{\pi}=-\frac{H^{\prime \prime}\left(\frac{1}{2} \pi\right)}{H\left(\frac{1}{2} \pi\right)} ;\left(\frac{2 K}{\pi}\right)^{2}=\frac{\Theta^{\prime \prime}(0)}{\Theta(0)}-\frac{H^{\prime \prime}\left(\frac{1}{2} \pi\right)}{H\left(\frac{1}{2} \pi\right)} \\
& \left(\frac{2 K k}{\pi}\right)^{2}=\frac{\Theta^{\prime \prime}(0)}{\Theta(0)}-\frac{\Theta^{\prime \prime}\left(\frac{1}{2} \pi\right)}{\Theta\left(\frac{1}{2} \pi\right)} ;\left(\frac{2 K k^{\prime}}{\pi}\right)^{2}=\frac{\Theta^{\prime \prime}\left(\frac{1}{2} \pi\right)}{\Theta\left(\frac{1}{2} \pi\right)}-\frac{H^{\prime \prime}\left(\frac{1}{2} \pi\right)}{H\left(\frac{1}{2} \pi\right)} \\
& \Theta^{\prime \prime}(x)=-4 q \frac{d \Theta(x)}{d q}, \quad H^{\prime \prime}(x)=-4 q \cdot \frac{d H(x)}{d q} \\
& \text { 5. }\left\{\begin{array}{l}
\frac{d \frac{2 K}{\pi}}{d q}=-\frac{1}{2 q} \cdot \frac{2 K}{\pi}\left\{\left(\frac{2 K k^{\prime}}{\pi}\right)^{2}-\frac{2 K}{\pi} \cdot \frac{2 E^{\prime}}{\pi}\right\} \\
\frac{d \frac{2 K k}{\pi}}{d q}=-\frac{1}{2 q} \cdot \frac{2 K k^{\prime}}{\pi}\left\{\left(\frac{2 K}{\pi}\right)^{2}-\frac{2 K}{\pi} \cdot \frac{2 E^{\prime}}{\pi}\right\} \\
\frac{d k}{d q}=\frac{1}{2 q} \cdot \frac{2 K k}{\pi} \cdot \frac{2 K}{\pi} \cdot \frac{2 E^{\prime}}{\pi} \cdot \\
\left.\frac{2 k^{\prime}}{\pi}\right)^{2} ; \frac{d k^{\prime}}{d q}=-\frac{1}{2 q} k^{\prime}\left(\frac{2 K k}{\pi}\right)^{2}
\end{array}\right.
\end{aligned}
$$




$$
\begin{aligned}
& \frac{1}{\sqrt{\left.k^{\prime}\right)}} \cdot \frac{d V^{\overline{k^{\prime}}}}{d q}=-\frac{1}{4 q}\left\{\frac{\Theta^{\prime \prime}(0)}{\Theta(0)}-\frac{\Theta^{\prime \prime}\left(\frac{1}{2} \pi\right)}{\Theta\left(\frac{1}{2} \pi\right)}\right\}=-\frac{1}{4 q} \cdot\left(\frac{2 K k}{\pi}\right)^{2} \\
& V^{k} \cdot \frac{d \frac{1}{\sqrt{k)}}}{d q}=-\frac{1}{4 q}\left\{\frac{\Theta^{\prime \prime}(0)}{\Theta(0)}-\frac{H^{\prime \prime}\left(\frac{1}{2} \pi\right)}{H\left(\frac{1}{2} \pi\right)}\right\}=-\frac{1}{4 q}\left(\frac{2 K^{k^{\prime}}}{\pi}\right)^{2} \\
& \left\{\begin{array}{l}
\frac{d \Delta \varphi}{d q}=-\frac{\Delta \varphi}{4 q} \cdot\left(\frac{2 K^{k}}{\pi}\right)^{2}-\frac{\Delta \varphi}{4 q}\left\{\frac{\Theta^{\prime \prime}\left(x+\frac{1}{2} \pi\right)}{\Theta\left(x+\frac{1}{2} \pi\right)}-\frac{\Theta^{\prime \prime}(x)}{\Theta(x)}\right\} \\
\frac{d \sin \varphi}{d q}=-\frac{\sin \varphi}{4 q} \cdot\left(\frac{2 K^{k}}{\pi}\right)^{2}-\frac{\sin \varphi}{4 q} \cdot\left\{\frac{H^{\prime \prime}(x)}{H(x)}-\frac{\Theta^{\prime \prime}(x)}{\Theta(x)}\right\} \\
\frac{d \cos \varphi}{d q}=-\frac{\cos \varphi}{4 q} \cdot\left(\frac{2 K}{\pi}\right)^{2}-\frac{\cos \varphi}{4 q} \cdot\left\{\frac{H^{\prime \prime}\left(x+\frac{1}{2} \pi\right)}{H\left(x+\frac{1}{2} \pi\right)}-\frac{\Theta^{\prime \prime}(x)}{\Theta(x)}\right\}
\end{array}\right. \\
& \text { 7. }\left\{\begin{array}{l}
\frac{d \Delta \varphi}{d \cdot x}=\Delta \varphi \cdot\left\{\frac{\Theta^{\prime}\left(x+\frac{1}{2} \pi\right)}{\Theta\left(x+\frac{1}{2} \pi\right)}-\frac{\Theta^{\prime}(x)}{\Theta(x)}\right\} \\
\frac{d \sin \varphi}{d x}=\sin \varphi \cdot\left\{\frac{H^{\prime}(x)}{H(x)}-\frac{\Theta^{\prime}(x)}{\Theta(x)}\right\} \\
\frac{d \cos \varphi}{d x}=\cos \varphi \cdot\left\{\frac{H^{\prime}\left(x+\frac{1}{2} \pi\right)}{H\left(x+\frac{1}{2} \pi\right)}-\frac{\Theta^{\prime}(x)}{\Theta(x)}\right\}
\end{array}\right.
\end{aligned}
$$

8. $\left\{\begin{array}{l}\frac{2 K}{\pi} Z(x) \cdot \frac{d \Delta \varphi}{d x}=\Delta \varphi \cdot\left\{\frac{\Theta^{\prime}\left(x+\frac{1}{2} \pi\right) \Theta^{\prime}(x)}{\Theta\left(x+\frac{1}{2} \pi\right) \Theta(x)}-\frac{\Theta^{\prime 2}(x)}{\Theta^{2}(x)}\right\} \\ \frac{2 K}{\pi} Z(x) \cdot \frac{d \sin \varphi}{d x}=\sin \varphi \cdot\left\{\frac{H^{\prime}(x) \Theta^{\prime}(x)}{H(x) \Theta(x)}-\frac{\Theta^{\prime 2}(x)}{\Theta^{2}(x)}\right\} \\ \frac{2 K}{\pi} Z(x) \cdot \frac{d \cos \varphi}{d x}=\cos \varphi \cdot\left\{\frac{H^{\prime}\left(x+\frac{1}{2} \pi\right) \Theta^{\prime}(x)}{H\left(x+\frac{1}{2} \pi\right) \Theta(x)}-\frac{\Theta^{\prime 2}(x)}{\Theta^{2}(x)}\right\}\end{array}\right.$

Im 37. Bande von Crelle's Journal habe ich folgende Formeln (33.) abgeleitet:

9.

$$
\left\{\begin{array}{l}
\frac{\Theta^{\prime \prime}(x)}{\Theta(x)}+\frac{\Theta^{\prime \prime}\left(x+\frac{1}{2} \pi\right)}{\Theta\left(x+\frac{1}{2} \pi\right)}-2 \frac{\Theta^{\prime}(x) \Theta^{\prime}\left(x+\frac{1}{2} \pi\right)}{\Theta(x) \Theta\left(x+\frac{1}{2} \pi\right)}=\frac{\Theta^{\prime \prime}\left(\frac{1}{2} \pi\right)}{\Theta\left(\frac{1}{2} \pi\right)}+-\frac{\Theta^{\prime \prime}(0)}{\Theta(0)} \\
\frac{\Theta^{\prime \prime}(x)}{\Theta(x)}+\frac{H^{\prime \prime}(x)}{H(x)}-2 \frac{\Theta^{\prime}(x) H^{\prime}(x)}{\Theta(x) H(x)}=\frac{H^{\prime \prime}\left(\frac{1}{2} \pi\right)}{H\left(\frac{1}{2} \pi\right)}+\frac{\Theta^{\prime \prime}\left(\frac{1}{2} \pi\right)}{\Theta\left(\frac{1}{2} \pi\right)} \\
\frac{\Theta^{\prime \prime}(x)}{\Theta(x)}+\frac{H^{\prime \prime}\left(x+\frac{1}{2} \pi\right)}{H\left(x+\frac{1}{2} \pi\right)}-2 \frac{\Theta^{\prime}(x) H^{\prime}\left(x+\frac{1}{2} \pi\right)}{\Theta(x) H\left(x+\frac{1}{2} \pi\right)}=\frac{H^{\prime \prime}\left(\frac{1}{2} \pi\right)}{H\left(\frac{1}{2} \pi\right)}+\frac{\Theta^{\prime \prime}(0)}{\Theta(0)}
\end{array}\right.
$$

Ferner ist

$$
\frac{\Theta^{\prime \prime}(x)}{\Theta(x)}-\frac{\Theta^{\prime 2}(x)}{\Theta^{2}(x)}=\frac{\Theta^{\prime \prime}(0)}{\Theta(0)}-\left(\frac{2 K k}{\pi}\right)^{2} \sin ^{2} \varphi
$$


Man multiplizire diese Gleichung mit 2 und ziehe sie von den Gleichungen (9) ab, so wird:

$$
\begin{aligned}
\frac{\Theta^{\prime \prime}\left(x+\frac{1}{2} \pi\right)}{\Theta\left(x+\frac{1}{2} \pi\right)}-\frac{\Theta^{\prime \prime}(x)}{\Theta(x)} & +2 \frac{\Theta^{\prime 2}(x)}{\Theta^{2}(x)}-2 \frac{\Theta^{\prime}(x) \Theta^{\prime}\left(x+\frac{1}{2} \pi\right)}{\Theta(x) \Theta\left(x+\frac{1}{2} \pi\right)} \\
& =2\left(\frac{2 K k}{\pi}\right)^{2} \sin ^{2} \varphi+\frac{\Theta^{\prime \prime}\left(\frac{1}{2} \pi\right)}{\Theta\left(\frac{1}{2} \pi\right)}-\frac{\Theta^{\prime \prime}(0)}{\Theta(0)} \\
\frac{H^{\prime \prime}(x)}{H(x)}-\frac{\Theta^{\prime \prime}(x)}{\Theta(x)} & +2 \frac{\Theta^{\prime 2}(x)}{\Theta^{2}(x)}-2 \frac{\Theta^{\prime}(x) H^{\prime}(x)}{\Theta(x) H(x)} \\
& =2\left(\frac{2 K k}{\pi}\right)^{2} \sin ^{2} \varphi+\frac{H^{\prime \prime}\left(\frac{1}{2} \pi\right)}{H\left(\frac{1}{2} \pi\right)}+\frac{\Theta^{\prime \prime}\left(\frac{1}{2} \pi\right)}{\Theta\left(\frac{1}{2} \pi\right)}-2 \frac{\Theta^{\prime \prime}(0)}{\Theta(0)} \\
\frac{H^{\prime \prime}\left(x+\frac{1}{2} \pi\right)}{H\left(x+\frac{1}{2} \pi\right)}-\frac{\Theta^{\prime \prime}(x)}{\Theta(x)} & +2 \frac{\Theta^{\prime 2}(x)}{\Theta^{2}(x)}-2 \frac{\Theta^{\prime}(x) H^{\prime}\left(x+\frac{1}{2} \pi\right)}{\Theta(x) H\left(x+\frac{1}{2} \pi\right)} \\
& =2\left(\frac{2 K k}{\pi}\right)^{2} \sin ^{2} \varphi+\frac{H^{\prime \prime}\left(\frac{1}{2} \pi\right)}{H\left(\frac{1}{2} \pi\right)}-\frac{\Theta^{\prime \prime}(0)}{\Theta(0)}
\end{aligned}
$$

welche Gleichungen mittelst der von $(6)$ und $(8)$ übergehn in:

$$
\begin{aligned}
& -2 q \frac{d \Delta \varphi}{d q}-\frac{d \Delta \varphi}{d x} \frac{2 K}{\pi} Z(x)=\left(\frac{2 K k}{\pi}\right)^{2} \sin ^{2} \varphi \Delta \varphi \\
& -2 g \frac{d \sin \varphi}{d q}-\frac{d \sin \varphi}{d x} \frac{2 K}{\pi} Z(x)=-\left(\frac{2 K k}{\pi}\right)^{2} \cos ^{2} \varphi \sin \varphi \\
& -2 q \frac{d \cos \varphi}{d q}-\frac{d \cos \varphi}{d x} \frac{2 K}{\pi} Z(x)=\left(\frac{2 K k}{\pi}\right)^{2} \sin ^{2} \varphi \cos \varphi
\end{aligned}
$$

Durch Differentiation erhält man:

$$
\begin{aligned}
& \int \frac{2 K}{\pi} Z(x) \cdot \frac{d \frac{\Delta \varphi}{1+b \sin \varphi}}{\frac{d x}{\sin \varphi}}=\frac{2 K}{\pi} Z(x) \cdot \frac{\frac{d \Delta \varphi}{d x}}{1+b \sin \varphi}-\frac{2 K}{\pi} Z(x) \frac{b \Delta \varphi \cdot \frac{d \sin \varphi}{d x}}{(1+b \sin \varphi)^{2}} \\
& \text { 11. }\left\{\frac{2 K}{\pi} Z(x) \cdot \frac{d \frac{\sin \varphi}{1+b \sin \varphi}}{d x}=\frac{2 K}{\pi} Z(x) \cdot \frac{\frac{d \sin \varphi}{d x}}{1+b \sin \varphi}-\frac{2 K}{\pi} Z(x) \frac{b \sin \varphi \cdot \frac{d \sin \varphi}{d x}}{(1+b \sin \varphi)^{2}}\right. \\
& \int \frac{2 K}{\pi} Z(x) \cdot \frac{d \frac{\cos \varphi}{1+b \sin \varphi}}{d x}=\frac{2 K}{\pi} Z(x) \cdot \frac{\frac{d \cos \varphi}{d x}}{1+b \sin \varphi}-\frac{2 K}{\pi} Z(x) \frac{b \cos \varphi \cdot \frac{d \sin \varphi}{d x}}{(1+b \sin \varphi)^{2}} \\
& \text { 12. }\left\{\begin{array}{l}
\frac{d \frac{\Delta \varphi}{1+b \sin \varphi}}{d q}=\frac{\frac{d \Delta \varphi}{d q}}{1+b \sin \varphi}-\frac{\Delta \varphi \sin \varphi \cdot \frac{d b}{d q}}{(1+b \sin \varphi)^{2}}-\frac{b \Delta \varphi \cdot \frac{d \sin \varphi}{d q}}{(1+b \sin \varphi)^{2}} \\
\frac{d \sin \varphi}{1+b \sin \varphi}=\frac{\frac{d \sin \varphi}{d q}}{d q}-\frac{\sin ^{2} \varphi \cdot \frac{d b}{d q}}{(1+b \sin \varphi}-\frac{b \sin \varphi \cdot \frac{d \sin \varphi}{d q}}{(1+b \sin \varphi)^{2}} \\
\frac{d+\cos \varphi}{1+b \sin \varphi} \\
\frac{d q}{d q}=\frac{\frac{d \cos \varphi}{d \eta}}{1+b \sin \varphi}-\frac{\cos \varphi \sin \varphi \cdot \frac{d b}{d q}}{(1+b \sin \varphi)^{2}}-\frac{b \cos \varphi \cdot \frac{d \sin \varphi}{d q}}{(1+b \sin \varphi)^{2}}
\end{array}\right.
\end{aligned}
$$


Multiplizirt man das System (12) mit $2 q$, addirt (11) hinzu, so heben sich vermöge der Gleichungen (10) die meisten Glieder der rechten Seite fort und es bleibt:

13.

$$
\begin{aligned}
& \frac{2 K}{\pi} Z(x) \frac{d \frac{\Delta \varphi}{1+b \sin \varphi}}{d x}+2 q \frac{d \frac{\Delta \varphi}{1+b \sin \varphi}}{d q}= \\
& -2 q \frac{\Delta \varphi \sin \varphi \frac{d b}{d q}}{(1+b \sin \varphi)^{2}}+\frac{\left(\frac{2 K k}{\pi}\right)^{2} \Delta \varphi \sin \varphi(b+\sin \varphi)}{(1+b \sin \varphi)^{2}} \\
& \frac{2 K}{\pi} Z(x) \frac{d \frac{\sin \varphi}{1+b \sin \varphi}}{d x}+2 q \frac{d \frac{\sin \varphi}{1+b \sin \varphi}}{d q}= \\
& -2 q \frac{\sin ^{2} \varphi \frac{d b}{d q}}{(1+b \sin \varphi)^{2}}-\frac{\left(\frac{2 K k}{\pi}\right)^{2} \cos ^{2} \varphi \cdot \sin \varphi}{(1+b \sin \varphi)^{2}} \\
& \frac{2 K}{\pi} Z(x)-\frac{d \frac{\cos \varphi}{1+b \sin \varphi}}{d x}+2 q \frac{d \frac{\cos \varphi}{1+b \sin \varphi}}{d q}= \\
& -2 q \frac{\cos \varphi \sin \varphi \frac{d b}{d q}}{(1+b \sin \varphi)^{2}}-\frac{\left(\frac{2 K k}{\pi}\right)^{2} \cos \varphi \sin \varphi(b+\sin \varphi)}{(1+b \sin \varphi)^{2}}
\end{aligned}
$$

oder

$$
\begin{gathered}
\frac{2 K}{\pi} Z(x) \frac{d \frac{\Delta \varphi}{1+b \sin \varphi}}{d x}= \\
-2 q \frac{d \frac{\Delta \varphi}{1+b \sin \varphi}}{d q}-\frac{2 q \Delta \varphi \sin \varphi \frac{d b}{d q}+\left(\frac{2 K k}{\pi}\right)^{2} \Delta \varphi \sin \varphi\{b+\sin \varphi\}}{(1+b \sin \varphi)^{2}}
\end{gathered}
$$$$
\frac{2 K}{\pi} Z(x) \frac{d \frac{\sin \varphi}{1+b \sin \varphi}}{d x}=
$$

14.

$$
\begin{aligned}
& -2 q \frac{d \frac{\sin \varphi}{1+b \sin \varphi}}{d q}-\frac{2 q \sin ^{2} \varphi \frac{d b}{d q}-\left(\frac{2 K k}{\pi}\right)^{2} \sin \varphi \cos ^{2} \varphi}{(1+b \sin \varphi)^{2}} \\
& \frac{2 K}{\pi} Z(x) \frac{d \frac{\cos \varphi}{1+b \sin \varphi}}{d x}= \\
& -2 q \frac{d \frac{\cos \varphi}{1+b \sin \varphi}}{d q}-\frac{2 q \cos \varphi \sin \varphi \frac{d b}{d q}+\left(\frac{2 \pi k}{\pi}\right)^{2} \cos \varphi \sin \varphi(b+\sin \varphi)}{(1+b \sin \varphi)^{2}}
\end{aligned}
$$


Dividirt man die Gleichungen 14 respective mit:

so wird:

$$
\frac{\Delta \varphi}{1+b \sin \varphi}, \quad \frac{\sin \varphi}{1+b \sin \varphi}, \quad \frac{\cos \varphi}{1+b \sin \varphi}
$$

15.

$$
\begin{aligned}
& \int \frac{2 K}{\pi} Z(x) \frac{d \log \frac{\Delta \varphi}{1+b \sin \varphi}}{d x}= \\
& -2 q \frac{d \log \frac{\Delta \varphi}{1+b \sin \varphi}}{d q}-\frac{2 q \sin \varphi \frac{d b}{d q}+\left(\frac{2 \pi k}{\pi}\right)^{2} \sin \varphi\{b+\sin \varphi\}}{1+b \sin \varphi} \\
& \frac{2 K}{\pi} Z(x) \frac{d \log \frac{\sin \varphi}{1+b \sin \varphi}}{d x}= \\
& -2 q \frac{d \log \frac{\sin \varphi}{1+b \sin \varphi}}{d q}-\frac{2 q \sin \varphi \frac{d b}{d q}-\left(\frac{2 K k}{\pi}\right)^{2} \cos ^{2} \varphi}{1+b \sin \varphi} \\
& \frac{2 K}{\pi} Z(x) \frac{d \log \frac{\cos \varphi}{1+b \sin \varphi}}{d x}= \\
& -2 q \frac{d \log \frac{\cos \varphi}{1+b \sin \varphi}}{d q}-\frac{2 q \sin \varphi \frac{d b}{d q}+\left(\frac{2 \pi k}{\pi}\right)^{2} \sin \varphi(b+\sin \varphi)}{1+b \sin \varphi}
\end{aligned}
$$

Hierin setze man $b=0$, so folgt als specieller Fall:

16.

$$
\left\{\begin{array}{l}
\frac{2 K}{\pi} Z(x) \cdot \frac{d \log \Delta \varphi}{d x}=-2 q \frac{d \log \Delta \varphi}{d q}-\left(\frac{2 K k}{\pi}\right)^{2} \sin ^{2} \varphi \\
\frac{2 K}{\pi} Z(x) \cdot \frac{d \log \sin \varphi}{d x}=-2 q \frac{d \log \sin \varphi}{d q}+\left(\frac{2 K k}{\pi}\right)^{2} \cos ^{2} \varphi \\
\frac{2 K}{\pi} Z(x) \cdot \frac{d \log \cos \varphi}{d x}=-2 q \frac{d \log \cos \varphi}{d q}-\left(\frac{2 K k}{\pi}\right)^{2} \sin ^{2} \varphi
\end{array}\right.
$$

Diese Gleichungen in derselben Reihenfolge von den darüberstehenden abgezogen fübren alle auf dieselbe Formel:

$$
\begin{aligned}
& \text { 17. } \quad \frac{2 \pi}{\pi} Z(x) \cdot \frac{d \log \frac{1}{1+b \sin \varphi}}{d x}= \\
& -2 q \frac{d \log \frac{1}{1+b \sin \varphi}}{d q}-\frac{2 q \sin \varphi \frac{d b}{d q}+\left(\frac{2 \pi k}{\pi}\right)^{2} b \sin \varphi \cos ^{2} \varphi}{1+b \sin \varphi}
\end{aligned}
$$


Man multiplizire endlich diese Gleichung mit $m$, die Gleichung 16 der Reihe nach mit $r, s, t$, so giebt die Summe dieser 4 Gleichungen:

$$
\begin{aligned}
& \text { 18. } \quad \frac{2 K}{\pi} Z(x) \frac{d \frac{\Delta \varphi^{r} \sin \varphi^{s} \cos \varphi^{t}}{(1+b \sin \varphi)^{m}}}{d x}= \\
& -2 q \frac{d \frac{\Delta \varphi^{r} \sin \varphi^{s} \cos \varphi^{t}}{(1+b \sin \varphi)^{m}}}{d q}-\frac{\Delta \varphi^{\tau} \sin \varphi^{s} \cos \varphi^{l}}{(1+b \sin \varphi)^{m}}\left\{(r+t)\left(\frac{2 K k}{x}\right)^{2} \sin ^{2} \varphi-s\left(\frac{2 K k}{\pi}\right)^{2} \cos ^{2} \varphi\right. \\
& \left.+m \frac{2 q \frac{d b}{d q} \sin \varphi+\left(\frac{2 \pi k}{\pi}\right)^{2} b \sin \varphi \cos ^{2} \varphi}{1+b \sin \varphi}\right\}
\end{aligned}
$$

Hierin kann $r, s, t, m$ jeden beliebigen Werth annehmen. Dieses ist die gesuchte Gleichung, welche jede Multiplikation einer ganzen oder gebrochenen Funktion mit dem Integral der 2ten Gattung auf eine Differentiation nach $q$ und auf eine Differentiation und Integration nach $x$ zurückführt. Denn wird

$$
\frac{\Delta \varphi^{r} \sin \varphi^{s} \cos \varphi^{\ell}}{(1+b \sin \varphi)^{m}}
$$

mit $U$ bezeichnet, so ist

$$
\frac{d\left\{\frac{2 K}{\pi} Z(x) \cdot U\right\}}{d x}=\frac{2 K}{\pi} Z^{\prime}(x) \cdot U+\frac{2 \pi}{\pi} Z(x) \cdot \frac{d U}{d x}
$$

und es folgt, indem für $\frac{2 K}{\pi} Z^{\prime}(x)$ sein Werth gesetzt wird:

$$
\begin{gathered}
-2 q \frac{d U}{d q}-U\left\{\frac{2 K}{\pi} \cdot \frac{2 E^{\prime}}{\pi}-\left(\frac{2 K}{\pi}\right)^{2}+(r+t+1)\left(\frac{2 K k}{\pi}\right)^{2} \sin ^{2} \varphi-s\left(\frac{2 K k}{\pi}\right)^{2} \cos ^{2} \varphi\right. \\
\left.+m \frac{2 q \frac{d b}{d q} \sin \varphi+\left(\frac{2 K k}{\pi}\right)^{2} b \sin \varphi \cos ^{2} \varphi}{1+b \sin \varphi}\right\}
\end{gathered}
$$

durch Integration nach $x$ erhält man das verlangte Produkt. 